\title{
Efficacy of Antimicrobial Lauric Arginate against Listeria monocytogenes on Stainless Steel Coupons
}

\author{
Jasdeep K. Saini, Miguel A. Barrios, James L. Marsden, Kelly J. K. Getty, Daniel Y. C. Fung \\ Department of Animal Sciences and Industry, Kansas State University, Manhattan, USA \\ Email: jasdeep@ksu.edu
}

Received December 29, 2012; revised January 30, 2013; accepted February 20, 2013

\begin{abstract}
Commercially processed foods become contaminated with Listeria monocytogenes in post-processing environments where favorable conditions help the bacteria thrive. The US Food and Drug Administration has approved Lauric arginate (LAE) as generally recognized as safe (GRAS) for certain food applications. This study evaluated the efficacy of Mirenat-N (LAE dissolved in food-grade propylene glycol) against L. monocytogenes on food contact surfaces. A three-strain cocktail of $L$. monocytogenes was used to inoculate 24 polished stainless steel coupons with three treatments, $100 \mathrm{ppm}$ and $200 \mathrm{ppm}$ solutions of LAE and water (control); two sub-treatments of high (6 $\log \mathrm{CFU} / \mathrm{ml})$ and low (4 $\log$ CFU/ml) inoculum levels; and two contact times of 5 and $15 \mathrm{~min}$. Attached bacteria were dislodged by vortexing coupons for $1 \mathrm{~min}$ with $20 \mathrm{~g}$ of $3-\mathrm{mm}$ solid glass beads in $10 \mathrm{ml}$ of $0.1 \%$ peptone diluent, and bacterial populations were calculated by plating onto modified oxford medium (MOX) and thin agar layer MOX (TALMOX). The $100 \mathrm{ppm}$ treatment showed average reductions of 1.38 and $2.57 \log \mathrm{CFU} /$ coupon at the low inoculum level and 0.37 and $0.62 \log$ $\mathrm{CFU} /$ coupon at high inoculum levels, after 5 and $15 \mathrm{~min}$ exposure, respectively. For $200 \mathrm{ppm}$ at the high inoculum level, 1.23 and $1.88 \log$ CFU/coupon reductions were seen for 5 and $15 \mathrm{~min}$, respectively; the low inoculum level at 5 and 15 min exposure showed reductions of $\leq 1.5 \log$ CFU/coupon. The $100 \mathrm{ppm}$ LAE treatment was more effective at low inoculum levels for 5 and 15 min contact times and may be used to control low levels of contamination of L. monocytogenes on food contact surfaces.
\end{abstract}

Keywords: Lauric Arginate; Listeria monocytogenes; Stainless Steel Coupons

\section{Introduction}

Bacteria have been shown to enter foods as a result of contact with contaminated surfaces [1]. Pathogenic bacteria can survive cleaning and disinfection of equipment surfaces in food processing environments, thus increasing the risks associated with transmission of disease [2,3]. Clear evidence suggests that contamination of commercially processed food products occurs with $L$. monocytogenes not because the organism is able to survive the processing operation, but because of contact with postprocessing environments that include food and non-food contact surfaces. Moisture plays an important role in survival and attachment of bacterial cells to different surfaces. Processing plant environments may easily become niches for bacterial cell development [4].

Sanitizers and cleaners are continuously evaluated for their use in food processing environments, and L. monocytogenes has shown sensitivity to several sanitizing agents. Research has shown that chlorine-based, iodinebased, acid anionic, and quaternary ammonium-based sanitizers used at concentrations of $100 \mathrm{ppm}, 25-45 \mathrm{ppm}$, $200 \mathrm{ppm}$, and $100-200 \mathrm{ppm}$, respectively, were effec- tive against $L$. monocytogenes $[5,6]$. Code of Federal Regulations Title 21 section 178.1010, Sanitizing solutions, defines the use of these sanitizers on finished product contact surfaces at $200 \mathrm{ppm}$ levels without requiring subsequent water rinse, except iodine-based sanitizers, where the maximum level is $25 \mathrm{ppm}$ [7].

Lauric arginate (LAE) is a novel antimicrobial that has been approved by the US Food and Drug Administration as generally recognized as safe (GRAS) for certain food applications $[8,9]$. Lauric arginate acts on cytoplasmic membranes of microorganisms to disrupt normal metabolic processes without causing cell lysis. The antimicrobial activity of LAE is attributed to its ability to alter cytoplasmic membranes of microorganisms [10]. In addition, LAE has a low oil-water equilibrium partition coefficient, which means it tends to concentrate in the aqueous phase of the products where most of the bacterial action occurs [8]. Because it is a cationic surfactant, LAE may interact strongly with other anionic or hydrophobic groups on a contact surface, thus defining its practical applications. Several studies have indicated reduction in bacterial populations of $L$. moncytogenes on the surface 
upon contact with LAE [6,11-13]. The objective of this study was to evaluate the efficacy of Mirenat-N, LAE dissolved in food-grade propylene glycol, against L. monocytogenes on stainless steel food product contact surfaces.

\section{Materials and Methods}

\subsection{Bacterial Cultures and Inoculum Preparation}

The bacterial cultures used in this study were obtained from the American Type Culture Collection (Rockville, MD) and included three strains of Listeria monocytogenes (ATCC 19115, 19113, 19112). The source of ATCC 19112 was spinal fluid from a man in Scotland, and ATCC 19115 (serotype 4b) and ATCC 19113 were human isolates. To prepare the inoculum, cultures were grown individually in $9 \mathrm{ml}$ of tryptic soy broth (TSB, Difco; Franklin Lakes, NJ) for $24 \mathrm{~h}$ at $35^{\circ} \mathrm{C}$. For inoculation purposes, each strain was combined into a single mixed culture suspension in phosphate buffer. The combined suspension was serially diluted to prepare two inoculum levels to inoculate the stainless steel surfaces. Targeted low (4 log CFU/ml) and high (6 log CFU/ml) levels of inoculum were prepared by serially diluting the culture suspension, then plated onto modified oxford medium agar (MOX, Oxoid, UK) and thin agar layer MOX agar (TALMOX), then incubated at $35^{\circ} \mathrm{C}$ for $24 \mathrm{~h}$ to confirm target levels.

\subsection{Preparation of Food Contact Surfaces}

Twenty-four polished stainless steel coupons (\#316 finish, $6.4 \times 1.9 \times 0.7 \mathrm{~cm}$ ) were initially cleaned using Sparkleen detergent (pH 9.5 - 10 in solution; Fisher Scientific, Hampton, New Hampshire), then autoclaved.

\subsection{Inoculation of Samples}

Two groups of eight coupons were inoculated with two different levels of inoculum (6 coupons to a level of attachment of $10^{4}$ and 6 coupons to a level of attachment of $10^{6}$ ) by dipping the coupons in the three-strain cocktail of L. monocytogenes for $1 \mathrm{~min}$ and allowing them to dripdry on racks for $30 \mathrm{~min}$.

\subsection{Treatment of Samples}

Solutions, $100 \mathrm{ppm}$ and $200 \mathrm{ppm}$, were prepared according to the labeled instructions of the manufacturer (Vedeqsa, Inc., Spain). After inoculation, one coupon from each group of 6 coupons was randomly assigned to each treatment as shown in Figure 1. Treatment application for hanging coupons (one from each group) was performed simultaneously with a spray bottle to apply the antimicrobial solution on both sides of the coupon. The first paired coupon was removed from each treatment or control solution after $5 \mathrm{~min}$ of exposure to the antimicrobial, and the second paired coupon was removed after 15 min of exposure.

\subsection{Recovery and Enumeration}

Each coupon was individually placed in a $50 \mathrm{ml}$ conical tube with $20 \mathrm{~g}$ of $3 \mathrm{~mm}$ sterile solid glass beads (Kimble Chase, Vineland, NJ) and $15 \mathrm{ml}$ of sterile $0.1 \%$ phosphate buffer (Difco, Franklin Lakes, NJ). Bacteria attached to the coupon were dislodged by vortexing for 1 min. Serial dilutions were prepared from the coupon wash suspension in $0.1 \%$ peptone diluent and spreadplated $(0.1 \mathrm{ml})$ onto MOX agar (Oxoid, UK) and thin agar layer MOX agar (TALMOX) [14]. These plates were incubated at $35^{\circ} \mathrm{C}$ for $48 \mathrm{~h}$, and recovered populations of $L$. monocytogenes were calculated and reported

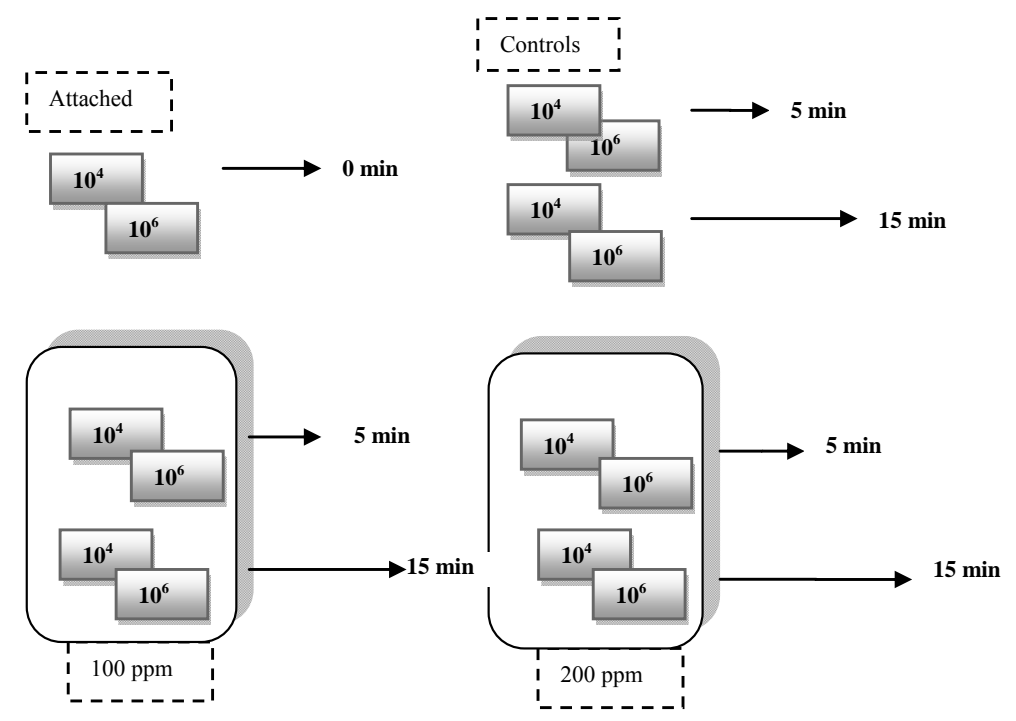

Figure 1. Schematic of treatment of stainless steel coupons with lauric arginate (LAE). 
as $\log \mathrm{CFU} /$ coupon. Three replications of the experimental set were performed.

\subsection{Statistical Analysis}

Listeria monocytogenes population data were analyzed using PROC MIXED in SAS version 9.0 (SAS Institute Inc., Cary, NC). Least square means $(\mathrm{P}<0.05)$ were used to compare interactions between treatment, inoculum level, and time of treatment. Fixed effects for statistical analysis were treatment, inoculum level, and time, and the random effect was replication.

\section{Results and Discussion}

The initial populations of $L$. monocytogenes on stainless steel coupons for high and low levels of inoculums were approximately $6 \log$ CFU/coupon and $4 \log$ CFU/coupon, respectively, as enumerated on MOX plates. Listeria monocytogenes populations differed $(\mathrm{P}<0.05)$ due to treatment $\times$ inoculum interaction. No difference $(\mathrm{P}>0.05)$ was observed between bacterial growth on MOX and TALMOX media, which indicated that observed reductions were due to a full lethality effect of LAE. No differences $(P>0.05)$ were observed when comparing the two treatments (100 ppm LAE vs. $200 \mathrm{ppm}$ LAE) tested after 5 min exposure (Figure 2). For 15 min contact time, $100 \mathrm{ppm}$ LAE and $200 \mathrm{ppm}$ LAE differed from each other $(\mathrm{P}<0.05)$ and the control (Figure 2).

For the $100 \mathrm{ppm}$ LAE treatment at the low inoculum level, 2.67 and $1.85 \log$ CFU/coupon of L. monocytogenes populations were recovered after 5 and $15 \mathrm{~min}$, respectively, whereas for the high inoculum level, 5.42 and $5.31 \log \mathrm{CFU} /$ coupon of L. monocytogenes populations were recovered after 5 and $15 \mathrm{~min}$, respectively

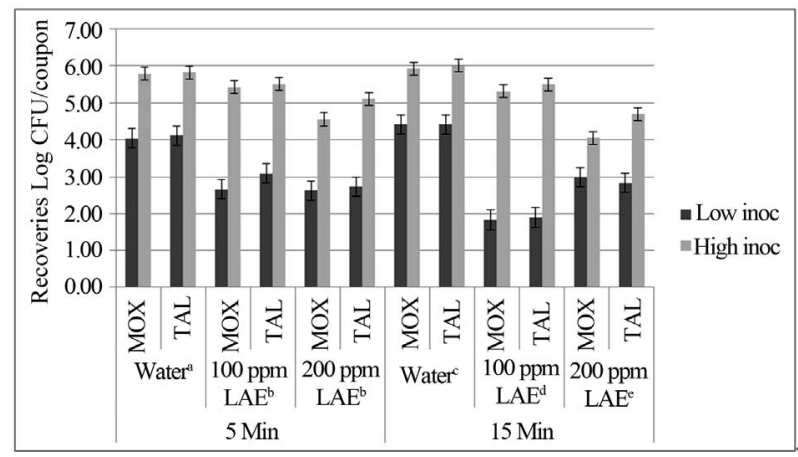

Figure 2. Mean and standard error $(n=3)$ of Listeria monocytogenes populations (log $\mathrm{CFU} / \mathrm{coupon})$ recovered on modified oxford medium (MOX) and thin agar layer MOX (TALMOX) from stainless steel coupons at high and low inoculum levels for 5 and 15 min treatments with water (positive control), $100 \mathrm{ppm}$ lauric arginate (LAE), and 200 ppm LAE solution. ${ }^{\mathrm{ab}}$ Indicate differences $(P<0.05)$ between treatments at $5 \mathrm{~min}$ and ${ }^{\text {cde }}$ indicate differences $(\mathrm{P}<$ 0.05) between treatments at $15 \mathrm{~min}$.
(Figure 2). For the $200 \mathrm{ppm}$ LAE treatment at the low inoculum level, 2.64 and $2.99 \log$ CFU/coupon bacterial populations were recovered after 5 and $15 \mathrm{~min}$, respectively, but for the high inoculum level, 4.55 and $4.05 \log$ $\mathrm{CFU} /$ coupon bacterial populations were recovered from stainless steel coupons after 5 and $15 \mathrm{~min}$, respectively (Figure 2). The $100 \mathrm{ppm}$ LAE treatment showed mean reductions of $1.38 \log \mathrm{CFU} /$ coupon after $5 \mathrm{~min}$ exposure and $2.57 \log \mathrm{CFU} /$ coupon after 15 min (Figure 3). This treatment appeared to be more effective for low contamination levels (low inoculum) than high concentration levels, where only 0.37 and $0.62 \log \mathrm{CFU} /$ coupon reductions in bacterial populations were observed for 5 and 15 min exposure, respectively.

At high inoculum levels, the $200 \mathrm{ppm}$ LAE treatment showed better lethality than the $100 \mathrm{ppm}$ LAE treatment with $1.23 \log \mathrm{CFU} /$ coupon reductions after $5 \mathrm{~min}$ and $1.88 \log$ CFU/coupon reductions in $15 \mathrm{~min}$ (Figure 3).

This interaction was expected, because as the concentration of the antimicrobial increases with the exposure time, lethality also increases.

Under the USDA/FSIS Listeria monocytogenes final rule, alternative 3 is control by sanitation, in which rigid sanitation control measures are to be incorporated into the production establishment's Hazard Analysis Critical Control Point (HACCP) plan, Sanitation Standard Operating Procedures (SSOP), or prerequisite program [15]. An effective cleaning and sanitation program is required. Cleaning is complete removal of food soil with the use of chemical compounds with appropriate detergent properties under recommended conditions, whereas sanitizing is the act of maintaining a clean environment by means of a physical or chemical agent. Sanitizer on food contact surface by definition is required to bring about a $5 \log$ reduction after a $30 \mathrm{~s}$ contact time [16]. Lauric arginate tested at 100 and $200 \mathrm{ppm}$ concentrations showed a

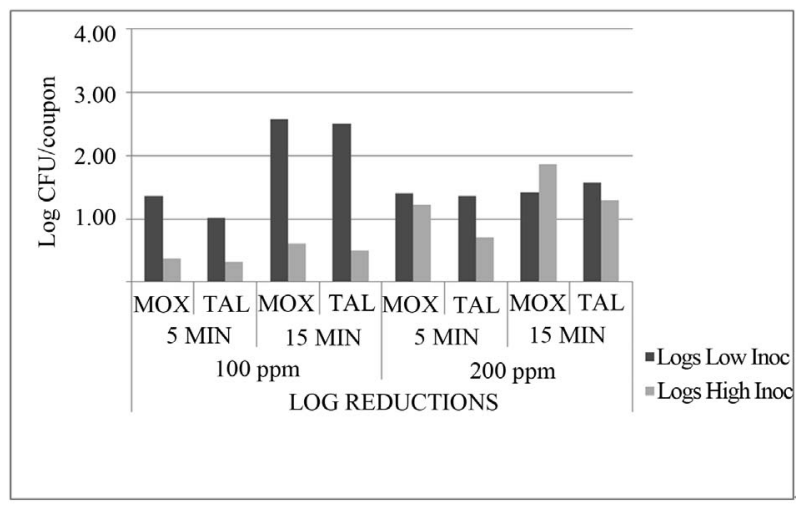

Figure 3. Reductions $(\mathbf{n}=3)$ in Listeria monocytogenes populations (log CFU/coupon) on modified oxford medium (MOX) and thin agar layer MOX (TALMOX) on stainless steel coupons due to treatment with 100 ppm and 200 ppm lauric arginate. 
maximum of $2.5 \log \mathrm{CFU} /$ coupon at $100 \mathrm{ppm}$ concentration with exposure time of $15 \mathrm{~min}$ at low bacterial concentration levels of $3 \log \mathrm{CFU} /$ coupon; therefore, it does not meet the requirements of being either a cleaner or sanitizer when used alone.

Given that LAE is labeled as GRAS, it may be used successfully as part of an integrated cleaning and sanitation program. Further research is needed to determine its efficacy against biofilms formed by L. monocytogenes in food processing environments.

\section{Acknowledgements}

This is contribution No. 13-045-J from the Kansas Agricultural Experiment Station.

\section{REFERENCES}

[1] P. J. Edington, H. Gibso, J. T. Holah, P. S. Handley and P. Gilbert, "The Influence of Substratum Properties on Attachment of Bacterial Cells," Colloids and Surfaces B, Vol. 5, No. 3-4, 1995, pp. 153-159. doi:10.1016/0927-7765(95)01219-9

[2] J. W. Austin and G. Bergeron, "Development of Bacterial Biofilms in Dairy Processing Lines," Journal of Dairy Research, Vol. 62, No. 3, 1995, pp. 509-549. doi:10.1017/S0022029900031204

[3] D. G. Dunsmore, A. Twomey, W. G. Whittlestone and H. W. Morgan, "Design and Performance of Systems for Cleaning Product-Contact Surfaces of Food EquipmentA Review," Journal of Food Protection, Vol. 44, No. 3, 1981, pp. 220-240.

[4] R. E. Faust and D. A. Gabis, "Controlling Microbial Growth in Food Processing Environments," Food Technology, Vol. 42, No. 12, 1988, pp. 81-82.

[5] J. A. Lopes, "Evaluation of Dairy and Food Plant Sanitizers against Salmonella Typhimurium and Listeria monocytogenes," Journal of Dairy Science, Vol. 69, No. 11, 1986, pp. 2791-2796. doi:10.3168/jds.S0022-0302(86)80731-7

[6] R. Orth and H. Mrozek, "Is the Control of Listeria, Campylobacter, and Yersinia a Disinfection Problem?" Fleischwirtschaft, Vol. 69, No. 10, 1989, pp. 1575-1576.

[7] Code of Federal Regulations (CFR), "Sanitizing Solutions," 2011.

http://www.gpo.gov/fdsys/pkg/CFR-2011-title21-vol3/pd
f/CFR-2011-title21-vol3-sec178-1010.pdf

[8] G. Bakal and A. Diaz, "The Lowdown on Lauric Arginate: Food Antimicrobial Hammers Away at Plasma Membrane, Disrupting a Pathogen's Metabolic Process," Food Quality, Vol. 12, No. 1, 2005, pp. 54-61.

[9] D. Benford, R. Harrison, J. Larsen and M. DiNovi, "Safety Evaluation of Certain Food Additives: Ethyl Lauroyl Arginate," World Health Organization, Geneva, 2009.

[10] E. Rodríguez, J. Seguer, X. Rocabayera and A. Manresa, "Cellular Effects of Monohydrochloride of L-Arginine, N-Lauroyl Ethylester (LAE) on Exposure to Salmonella Typhimurium and Staphylococcus aureus," Journal of Applied Microbiology, Vol. 96, No. 5, 2004, pp. 903-912. doi:10.1111/j.1365-2672.2004.02207.x

[11] A. C. S. Porto-Fett, S. G. Campano, J. L. Smith, A. Oser, B. Shoyer, J. E. Call and J. B. Luchansky, "Control of Listeria monocytogenes on Commercially-Produced Frankfurters Prepared with and without Potassium Lactate and Sodium Diacetate and Surface Treated with Lauric Arginate Using the Sprayed Lethality in Container (SLIC) Delivery Method," Meat Science, Vol. 85, No. 2, 2010, pp. 312-318. doi:10.1016/i.meatsci.2010.01.020

[12] P. J. Toarmina and W. J. Dorsa, "Short-Term Bactericidal Efficacy of Lauric Arginate against Listeria monocytogenes Present on the Surface of Frankfurters," Journal of Food Protection, Vol. 72, No. 6, 2009, pp. 1216-1224.

[13] E. M. Martin, C. L. Griffis, K. L. S. Vaughn, C. A. O'Bryan, E. C. Friedly, J. A. Marcy, S. C. Ricke, P. G. Crandall and R. Y. Lary, "Control of Listeria monocytogenes by Lauric Arginate on Frankfurters Formulated with or without Lactate/Diacetate," Journal of Food Science, Vol. 74, No. 6, 2009, pp. 237-241. doi:10.1111/j.1750-3841.2009.01196.x

[14] D. H. Kang and D. Y. C. Fung, "Thin Agar Layer Method for Recovery of Heat-Injured Listeria monocytogenes," Journal of Food Protection, Vol. 62, No. 11, 2009, pp. 1346-1349.

[15] Food Safety and Inspection Service (FSIS), "Updated Compliance Guidelines to Control LM in Post-Lethality Exposed Ready-To-Eat Meat and Poultry Products," 2006. http://www.fsis.usda.gov/oppde/rdad/frpubs/97-013f/lm_r ule_compliance_guidelines_may_2006.pdf

[16] A. Parker, "Effective Cleaning and Sanitizing Procedures. JIFSAN Good Aquacultural Practices Program," 2007. http://www.jifsan.umd.edu/pdf/gaqps_en/09\%20GAqPs\% 20Manual\%20CleaningSanitation.pdf 